\title{
Large-cell neuroendocrine carcinoma of nasal cavity and paranasal sinuses after successful curative therapy: a case report and literature review
}

This article was published in the following Dove Press journal:

OncoTargets and Therapy

\author{
Qin Zhao'* \\ Jinlong Wei ${ }^{1, *}$ \\ Chengbin Zhang ${ }^{2}$ \\ Lingbin Meng ${ }^{3}$ \\ Bin Wangl \\ Ling Gao ${ }^{1, *}$ \\ Xin Jiang ${ }^{1} *$ \\ 'Department of Radiation Oncology, The \\ First Hospital of jilin University, \\ Changchun 13002I, People's Republic of \\ China; ${ }^{2}$ Department of pathology, the \\ First Hospital of Jilin University, \\ Changchun I3002I, People's Republic of \\ China; ${ }^{3}$ Department of Internal Medicine, \\ Florida Hospital, Orlando, FL 32804, USA \\ *These authors contributed equally to \\ this work
}

Correspondence: Ling Gao; Xin Jiang Department of Radiation Oncology, The First Hospital of Jilin University, 71 Xinmin Street, Changchun I3002I, People's Republic of China Tel +86 I58 04303772 ; $+8615804302750$

Email linggao2010@126.com; jiangx@jlu.edu.cn

\begin{abstract}
This report describes a 40-year-old male patient with symptoms affecting the nasal sinuses including nasal obstruction and olfactory anesthesia. Magnetic resonance imaging demonstrated that the tumor eroded the adjacent bone and bilateral frontal lobes. The biopsy sample of the left nasal mass was processed for both $H \& E$ staining and immunohistological staining for various markers. The final histological examination showed large-cell neuroendocrine carcinoma with poor differentiation. After a general evaluation, the patient was staged as cT4bN0M0. The patient was treated by combined radiotherapy and chemotherapy. The treatment yielded almost complete remission, and after 10 months of follow-up, no distant organ metastasis or recurrence in the primary tumor region was detected. We report this rare case and review the current literature of this tumor.
\end{abstract}

Keywords: nasal cavity and paranasal sinuses carcinoma, large-cell neuroendocrine carcinoma, pathology, radiotherapy

\section{Introduction}

Large-cell neuroendocrine carcinoma (LCNEC) is a rare epithelial neuroendocrine malignancy and is preferentially located in the gastrointestinal tract and pancreas. ${ }^{1}$ Cases of LCNEC have been reported in many other locations, including the thymus, gallbladder, prostate, larynx, salivary glands, nose, nasopharynx, tonsil, hard palate and mastoid. $^{2-5}$ However, LCNEC of the nasal cavity and paranasal sinuses is rarely reported.

Classification of nasal cavity and paranasal sinus carcinomas is manifold. The WHO published the extensively revised 4th edition of the Classification of Head and Neck Tumors in 2017. Although LCNEC was not previously acknowledged, the new edition recognizes small-cell neuroendocrine carcinoma and LCNECs as distinct types. ${ }^{6}$ Radiotherapy as a treatment option for nasal cavity and paranasal sinus carcinomas has been widely investigated, but no studies have reported its effects on LCNEC. We present the case of a male patient with LCNEC located in the nasal cavity and paranasal sinuses who underwent successful radiotherapy and chemotherapy and achieved a clinically complete recovery.

\section{Case report}

A 40-year-old male patient was initially diagnosed with nasal polyps at a local county hospital after he presented with symptoms of nasal obstruction and olfactory anesthesia. However, the symptoms significantly worsened within 1 month. The 
patient was thereafter admitted to the First Hospital of Jilin University. Contrast-enhanced magnetic resonance imaging (MRI) demonstrated that a tumor was located in the bilateral maxillary sinus, ethmoid sinus, frontal sinus, sphenoid sinus and left nasal cavity without enlarged lymph nodes in the bilateral neck. The maximum diameter of the tumor was $7.0 \times 5.2 \mathrm{~cm}$, and the tumor eroded the adjacent bones including the bilateral maxillary sinus medial wall, sieve plate, sphenoid sinus, frontal sinus wall and bilateral frontal lobes (Figure 1A). A biopsy of the patient's left nasal mass was performed. To characterize the cells, the biopsy sample was processed for both conventional $\mathrm{H} \& \mathrm{E}$ staining and immunohistological staining for various markers. The LCNEC was positive for Ki-67, CKpan, CgA, Syn and CD56. Additional stains for HMB45, S-100, Vimentin, EBER, CD20 and CD3 were negative. The final histological examination showed LCNEC with poor differentiation (Figure 2).

This patient did not undergo positron emission tomography/computed tomography (PET-CT) scan due to economic reasons. After a general evaluation, the patient was staged as cT4bN0M0 according to the staging system established by American Joint Committee on Cancer (AJCC) in 2010. The patient received one cycle of neoadjuvant chemotherapy (etoposide combined with nedaplatin, EP regimen); however, the symptoms did not improve significantly. So we decided to give concurrent radiochemotherapy after multidisciplinary consultation. External radiation therapy (RT) was administered with the TrueBeam linear accelerator using volumetric modulated arc therapy (VMAT). A total dose of 5,040 cGy with $180 \mathrm{cGy} /$ fraction was applied to the bilateral maxillary sinus, ethmoid sinus, frontal sinus, nasal cavity, and upper neck region (levels II-III), while a total of 6,996 cGy with $212 \mathrm{cGy} /$ fraction was applied to the carcinoma of the nasal cavity and paranasal sinuses. During RT, the EP regimen was administered in 2 cycles. Following concurrent radiochemotherapy, the patient was treated with three cycles of sequential adjuvant chemotherapy (EP regimen).

After chemoradiotherapy, the treatment led to almost complete remission (CR) as assessed with the Response Evaluation Criteria in Solid Tumors (RECIST 1.1) (Figure 1B), and no adverse reactions were reported. After 10 months of follow-up, no distant organ metastasis or recurrence in the primary tumor region was detected. The short-term treatment is an effective therapy, although the follow-up is only 10 months, and longterm follow-up work still needs to be continued.
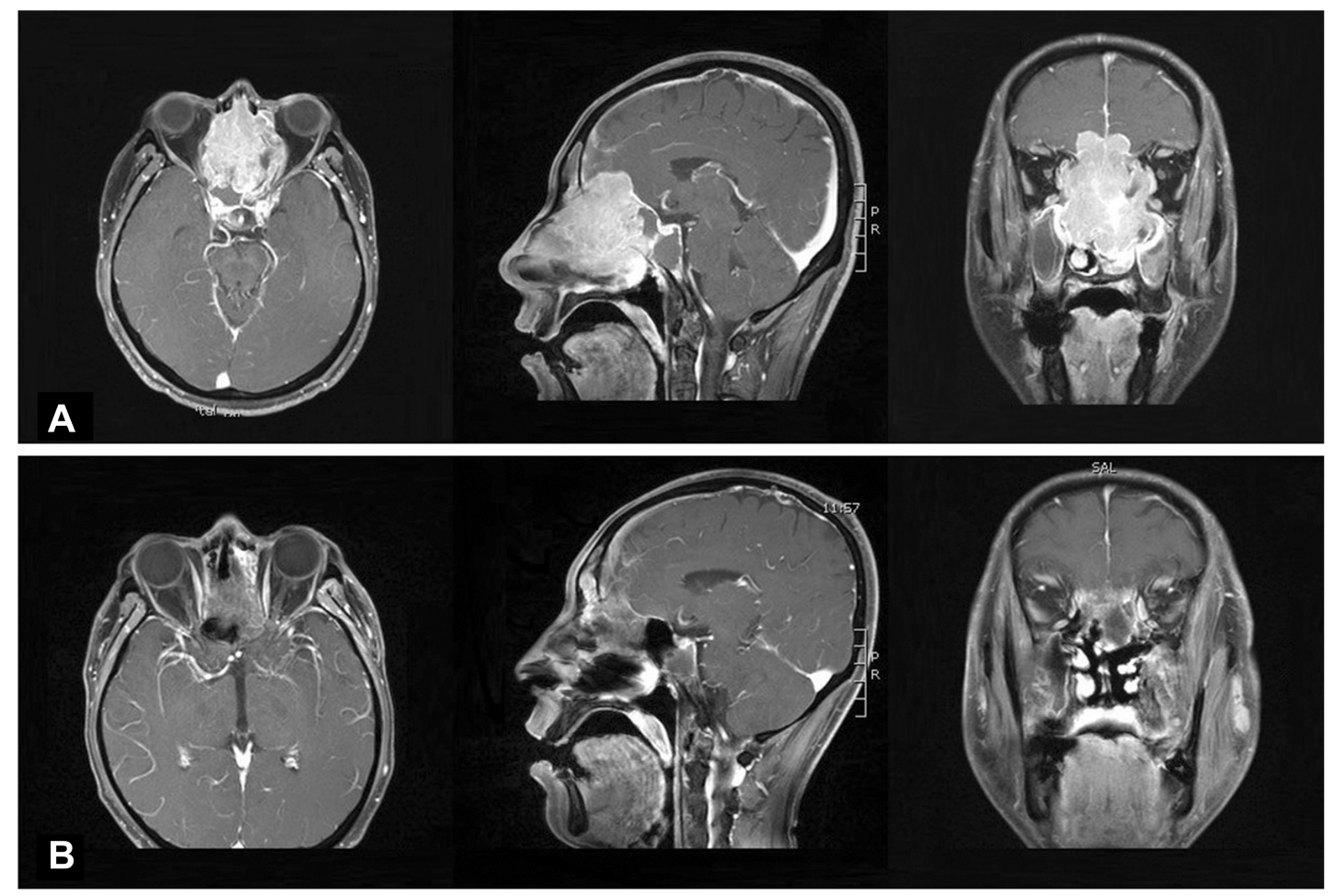

Figure I MRI of the nasal cavity and paranasal sinuses. (A) Contrast-enhanced MRI shows a huge tumor located in the nasal cavity and paranasal sinuses eroded not only the adjacent bone but also bilateral frontal lobes. (B) Contrast-enhanced MRI shows the tumor was almost completely disappeared I month after radiotherapy and chemotherapy. Abbreviation: MRI, magnetic resonance imaging. 

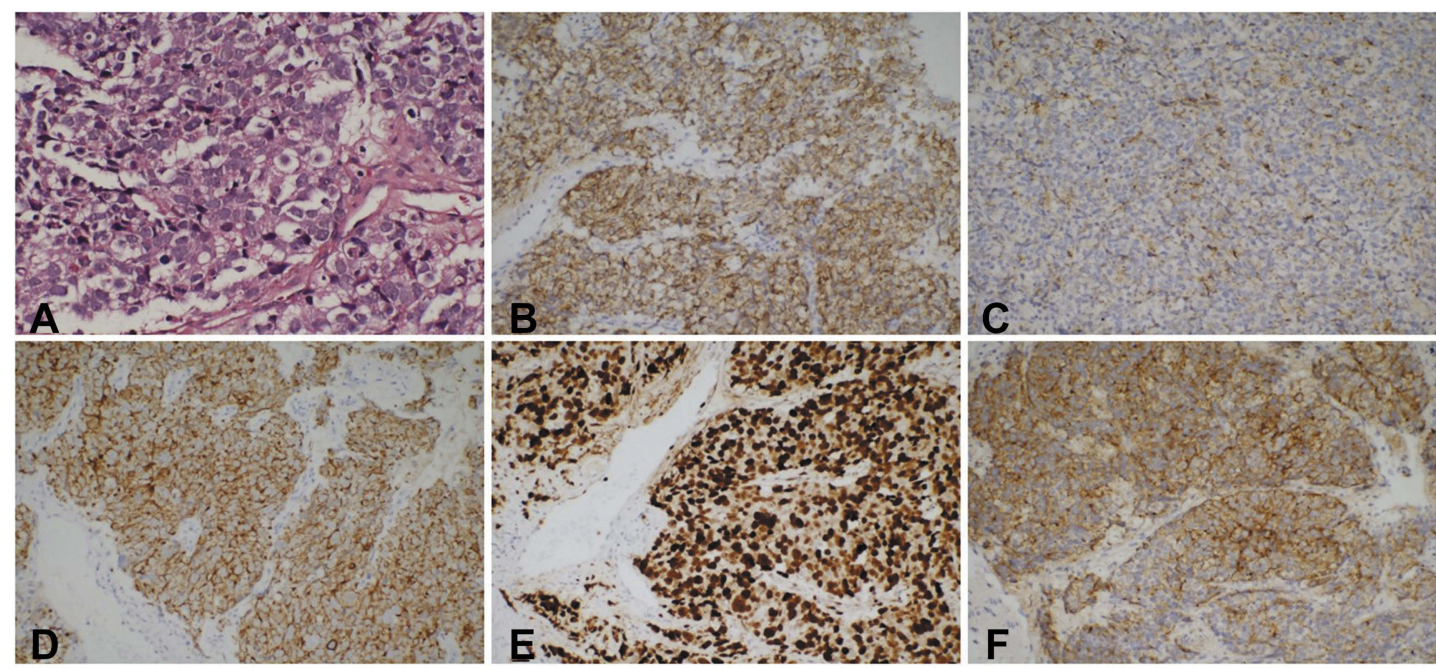

Figure 2 Histopathology of LCNEC. (A) H\&E staining: tumor cells in the subepithelial stroma showed nest infiltration. The cells are large in volume, rich in cytoplasm and vacuolated or eosinophilic and have a large nucleoplasmic ratio. The nucleus is round or elliptical, the chromatin is deeply stained, and the granules are coarse and granular, and the obvious eosinophilic nucleoli can be seen (400xmagnification). Immunohistological staining showing tumor positivity for CD56 (B), CgA (C), CKpan (D), Ki-67 (E) and Syn (F).

Abbreviation: LCNEC, large-cell neuroendocrine carcinoma.

\section{Discussion}

Sinonasal carcinomas are a rare group of malignant neoplasms that arise in the nasal cavity or its adjacent paranasal sinuses. Sinonasal carcinomas comprise $5 \%$ of head and neck cancers and fewer than $1 \%$ of all malignant tumors. ${ }^{7}$ The overall annual incidence of sinonasal carcinoma is 0.556 cases per 100,000 people with a male/female ratio of 1.8:1. The most common histologies are squamous cell carcinoma (51.6\%) and adenocarcinoma (12.6\%), whereas the most common primary sites are the nasal cavity (43.9\%) and maxillary sinus $(35.9 \%) .{ }^{8}$ The prognosis of patients with sinonasal cancer is generally poor and has not changed substantially over the last 3 decades. $^{8}$ Due to the development of multidisciplinary therapy, the 5-year survival rate of sinonasal carcinomas was significantly higher in 2010-2014 $(65 \%)$ than in $1980-1984(46 \%){ }^{9}$

Sinonasal neuroendocrine carcinoma (SNEC) is a rare and high-grade malignant epithelial neoplasm that is usually associated with a poor prognosis as well as immunohistochemical features of neuroendocrine differentiation. Tapan et $\mathrm{al}^{7}$ analyzed the clinical pathological characteristics and survival outcomes of SNEC and found that the overall 5-year diseasespecific survival (DSS) rate for SNEC was 50.8\%. Five-year survival analysis for SNEC by site revealed DSS rates of $80.7 \%, 59.2 \%, 34.5 \%$ and $33.0 \%$ for the sphenoid sinus, nasal cavity, maxillary sinus and ethmoid sinus, respectively.

SNEC is separated into small-cell and large-cell types. LCNEC is much rarer and presents unique challenges both in diagnosis and in treatment. The clinical presentations of sinonasal LCNEC include nasal obstruction, epistaxis, nasal drainage, facial mass and facial pain, and there is no clinical difference between both small-cell and largecell type. However, the large-cell subtype shows a better survival rate than that of the small cell. ${ }^{10}$ These symptoms overlap with other those of benign sinonasal diseases and often delay diagnosis and appropriate treatment. This patient was diagnosed with a locally advanced tumor that developed rapidly; however, it was not accompanied by the enlarged lymph nodes in the bilateral neck that are typical of this disease. LCNEC is a high-grade neuroendocrine neoplasm and generally associated with poor prognosis. However, tumor staging appears of limited value in predicting survival or selecting a treatment strategy. ${ }^{11}$ Although the patient was diagnosed with an advanced and rapidly developing tumor, chemoradiotherapy was an effective and successful treatment.

Histologically, LCNEC is characterized by 1) large, polygonal cells with coarse nuclear chromatin and prominent nucleoli, 2) high mitotic rate and frequent necrosis, 3) architectural patterns suggestive of neuroendocrine differentiation (organoid nests, trabeculae, rosettes and/or peripheral palisading) and 4) immunohistochemical evidence of neuroendocrine differentiation (ie, immunostaining with Syn, CKpan, CgA and/or CD56). ${ }^{12}$ Because there is not yet an immunohistochemical stain that can discriminate between small-cell neuroendocrine carcinoma (SCNEC) and LCNEC, a panel of three 
markers, BAI3, CDX2 and VIL1, was used for diagnosis. ${ }^{13}$ The critical distinction between SCNEC and LCNEC is not in the overall cell size but in the nuclear to cytoplasmic ratios ("high" in small cell and "lower" in large cell). ${ }^{14}$ The major feature that distinguishes LCNEC from atypical carcinoids is mitotic activity ( $>10 / 10$ high-power fields or $\left.2 \mathrm{~mm}^{2}\right)$ and necrosis, with LCNEC typically having more extensive necrosis and more pleomorphic cells with prominent nucleoli. ${ }^{15}$

CT and MRI are the most commonly used techniques to determine the location and local extension of SNEC. CT has several advantages in evaluating skeletal changes such as cortical erosion, destruction and thickening of bones. However, MRI is complementary to CT because it can effectively characterize soft tissue components and evaluate the extent of tumor invasion beyond the bony sinus walls. ${ }^{16}$ The utility of $\left[{ }^{18} \mathrm{~F}\right]$ fluorodeoxyglucose (FDG)$\mathrm{PET} / \mathrm{CT}$ imaging is not recommended for routine diagnosis and staging of head and neck cancer in most guidelines; ${ }^{17}$ however, it has been shown to be useful for the imaging of residual and recurrent tumors. ${ }^{18}$

Van der Laan et $\mathrm{al}^{11}$ found that the most important predictors of survival in SNEC are differentiation grade and the associated choice of treatment modality. Furthermore, they recommended that surgery should be the cornerstone of treatment, supplemented by radiotherapy in poorly differentiated subtypes. The optimal treatment of LCNEC is a complicated issue due to the low numbers of patients and the staggering amount of different histologies. Prospective studies are scarce; a search of the PubMed database revealed only a few case reports and small retrospective case series. The treatment of LCNEC is controversial and not yet standardized because there are no clinical trials that define the optimal treatment approach for either localized or advanced disease. Some suggest a multimodal appraoch to the treatment of sinonasal carcinomas which includes surgical resection, chemotherapy and radiotherapy.

Surgery is the preferred treatment in the majority of sinonasal carcinomas. Resection of tumors with clear margins leads to an excellent prognosis. However, this patient was diagnosed in an advanced state, and complete surgical resection would be difficult. Hanna et $\mathrm{al}^{19}$ retrospectively analyzed 120 patients who underwent endoscopic resection of sinonasal cancer between 1992 and 2007, and approximately 10\% of the tumors had an intracranial epicenter, most commonly around the olfactory groove. Tumors extended to or invaded the skull base in $20 \%$ and $11 \%$ of the patients, respectively.

The oncologic outcomes of patients with sinonasal carcinomas have been improved, likely due to more effective reconstruction using vascularized flaps and more effective adjuvant therapy, particularly intensity-modulated radiation therapy (IMRT). Radiotherapy may provide effective local control for patients with moderately differentiated NEC if resection is not feasible, while post-RT surgical resection can benefit patients with chemoresistant or radioresistant disease. $^{20}$ Postoperative RT was usually used in patients with high-grade tumors, advanced $\mathrm{T}$ stage, bone invasion, perineural spread, intracranial extension, dural or brain involvement or positive margins. Duprez et $\mathrm{al}^{21}$ retrospectively analyzed 130 patients with nonmetastatic sinonasal tumors who were treated with IMRT. In this study, we did not observe Grade 4 acute ocular toxicity, and neither radiationinduced blindness nor severe dry eye syndrome was observed in late toxicity. IMRT for sinonasal tumors should be considered as the standard radiotherapy for patients who are not surgically treated.

The patient received VMAT, a new RT treatment delivered using a continuous arc motion of the gantry with simultaneous variation of the multileaf collimator position, gantry speed and dose rate, with the ability to produce highly conformal plans in a short time. ${ }^{22}$ VMAT achieved a better conformity and more homogenous target coverage compared with IMRT. Additionally, VMAT has a faster delivery time and a lower number of monitor units. VMAT can also optimize the radiation dose to the tumor and reduce toxic effects to normal structures, particularly the lens.

There may be also a couple of limitations of the case report. The follow-up time of this case is very short. But LCNEC located in the nasal cavity and paranasal sinuses is rare, and we cannot find sufficient data for survival, local, regional or distant recurrences. A paper showed that the patients with LCNEC of prostate died of their disease within a short period of time after the detection of LCNEC. ${ }^{5}$ Another research reported that LCNEC represented an aggressive tumor type and has a poor prognosis, similar to that of small-cell carcinoma. ${ }^{2}$ Another limitation is that this patient did not receive the PET-CT scan before chemotherapy and radiotherapy due to economic reasons. We have done other examinations including history and physical, chest and abdominal CT, bone scan and local MRI, and results showed no obvious symptoms of distant metastasis. Based on those examinations, the patient was staged as cT4bN0M0.

\section{Conclusion}

LCNEC is a rare epithelial neuroendocrine malignancy generally associated with poor prognosis. However, staging appears of limited value in predicting survival. Due to the 
rarity of LCNEC, a general consensus on the standard therapy has not yet been established. Surgery is the preferred treatment, but radiochemotherapy is also safe and effective.

\section{Consent to publish}

Written informed consent was obtained from the patient for publication of this case report and the accompanying images. This report was approved by the Ethics Committee of The First Hospital of Jilin University.

\section{Acknowledgments}

This report was supported in part by grants from the Norman Bethne Program of Jilin University (2015225, to Ying Xin and 2015203, to Xin Jiang), the Education Department of Jilin Province Foundations (2016-448 to Xin Jiang), the Jilin Provincial Science and Technology Foundations (20180414039GH to Ying Xin and 20190201200JC to Xin Jiang) and the Health and Family Planning Commission of Jilin Province Foundations (2016Q034 to Ying Xin and 2015Q010 to Xin Jiang). Qin Zhao and Jinlong Wei are cofirst authors for this study.

\section{Disclosure}

The authors report no conflicts of interest in this work.

\section{References}

1. Klimstra DS, Modlin IR, Coppola D, Lloyd RV, Suster S. The pathologic classification of neuroendocrine tumors: a review of nomenclature, grading, and staging systems. Pancreas. 2010;39(6):707-712. doi:10.1097/MPA.0b013e3181ec124e

2. Lin D, Suwantarat N, Kwee S, Miyashiro M. Cushing's syndrome caused by an ACTH-producing large cell neuroendocrine carcinoma of the gallbladder. World $J$ Gastrointest Oncol. 2010;2(1):56-58. doi:10.4251/wjgo.v2.i1.56

3. Chetty R, Shah KA, Perez-Ordonez B. Large cell neuroendocrine carcinoma of the head and neck. Am J Surg Pathol. 2012;36 (7):1102-1103. doi:10.1097/PAS.0b013e318254e80e

4. Ogawa F, Iyoda A, Amano H, et al. Thymic large cell neuroendocrine carcinoma: report of a resected case - a case report. $J$ Cardiothorac Surg. 2010;5:115. doi:10.1186/1749-8090-5-115

5. Evans AJ, Humphrey PA, Belani J, van der Kwast TH, Srigley JR. Large cell neuroendocrine carcinoma of prostate: a clinicopathologic summary of 7 cases of a rare manifestation of advanced prostate cancer. Am J Surg Pathol. 2006;30(6):684-693.

6. Ye D, Wang Y, Li H, et al. Fibroblast growth factor 21 protects against acetaminophen-induced hepatotoxicity by potentiating peroxisome proliferator-activated receptor coactivator protein-1alpha-mediated antioxidant capacity in mice. Hepatology. 2014;60(3):977-989. doi:10.1002/hep. 27060
7. Patel TD, Vazquez A, Dubal PM, Baredes S, Liu JK, Eloy JA. Sinonasal neuroendocrine carcinoma: a population-based analysis of incidence and survival. Int Forum Allergy Rhinol. 2015;5 (5):448-453. doi:10.1002/alr.21497

8. Turner JH, Reh DD. Incidence and survival in patients with sinonasal cancer: a historical analysis of population-based data. Head Neck. 2012;34(6):877-885. doi:10.1002/hed.21830

9. Sjostedt S, Jensen DH, Jakobsen KK. Incidence and survival in sinonasal carcinoma: a Danish population-based, nationwide study from 1980 to 2014. Acta Oncol. 2018;57(9):1152-1158. doi:10.1080/ 0284186X.2018.1454603

10. Turri-Zanoni M, Maragliano R, Battaglia P, et al. The clinicopathological spectrum of olfactory neuroblastoma and sinonasal neuroendocrine neoplasms: refinements in diagnostic criteria and impact of multimodal treatments on survival. Oral Oncol. 2017;74:21-29. doi:10.1016/j.oraloncology.2017.09.010

11. van der Laan TP, Iepsma R, Witjes MJH, van der Laan BFAM, Plaat BEC, Halmos GB. Meta-analysis of 701 published cases of sinonasal neuroendocrine carcinoma: the importance of differentiation grade in determining treatment strategy. Oral Oncol. 2016;63:1-9. doi:10.1016/j.oraloncology.2016.10.002

12. Thompson ED, Stelow EB, Mills SE, Westra WH, Bishop JA. Large cell neuroendocrine carcinoma of the head and neck: a clinicopathologic series of 10 cases with an emphasis on HPV status. Am J Surg Pathol. 2016;40(4):471-478. doi:10.1097/PAS.0000000000000580

13. Bari MF, Brown H, Nicholson AG, et al. BAI3, CDX2 and VIL1: a panel of three antibodies to distinguish small cell from large cell neuroendocrine lung carcinomas. Histopathology. 2014;64 (4):547-556. doi:10.1111/his. 12278

14. Ferlito A, Strojan P, Lewis JS, Perez-Ordoñez B, Rinaldo A. Large cell neuroendocrine carcinoma of the head and neck: a distinct clinicopathologic entity. Eur Arch Otorhinolaryngol. 2014;271 (8):2093-2095. doi:10.1007/s00405-014-3090-7

15. Kusafuka K, Ferlito A, Lewis JS, et al. Large cell neuroendocrine carcinoma of the head and neck. Oral Oncol. 2012;48(3):211-215. doi:10.1016/j.oraloncology.2011.09.016

16. Eggesbø HB. Imaging of sinonasal tumours. Cancer Imaging. 2012;12:136-152. doi:10.1102/1470-7330.2012.0015

17. Bussink J, van Herpen CML, Kaanders JHAM, Oyen WJG. PET-CT for response assessment and treatment adaptation in head and neck cancer. Lancet Oncol. 2010;11(7):661-669. doi:10.1016/S14702045(09)70353-5

18. Fukui MB, Blodgett TM, Snyderman CH, et al. Combined PET-CT in the head and neck: part 2. Diagnostic uses and pitfalls of oncologic imaging. Radiographics. 2005;25(4):913-930. doi:10.1148/rg.254045136

19. Hanna E, DeMonte F, Ibrahim S, Roberts D, Levine N, Kupferman M. Endoscopic resection of sinonasal cancers with and without craniotomy: oncologic results. Arch Otolaryngol Head Neck Surg. 2009;135(12):1219-1224. doi:10.1001/archoto.2009.173

20. Likhacheva A, Rosenthal DI, Hanna E, Kupferman M, DeMonte F, El-Naggar AK. Sinonasal neuroendocrine carcinoma: impact of differentiation status on response and outcome. Head Neck Oncol. 2011;3:32. doi:10.1186/1758-3284-3-32

21. Duprez F, Madani I, Morbée L, et al. IMRT for sinonasal tumors minimizes severe late ocular toxicity and preserves disease control and survival. Int J Radiat Oncol Biol Phys. 2012;83(1):252-259. doi:10.1016/j.ijrobp.2011.06.1977

22. Mashhour K, Kamaleldin M, Hashem W. RapidArc vs conventional IMRT for head and neck cancer irradiation: is faster necessary better? Asian Pac J Cancer Prev. 2018;19(1):207-211. doi:10.22034/ APJCP.2018.19.1.207 


\section{Publish your work in this journal}

OncoTargets and Therapy is an international, peer-reviewed, open access journal focusing on the pathological basis of all cancers, potential targets for therapy and treatment protocols employed to improve the management of cancer patients. The journal also focuses on the impact of management programs and new therapeutic agents and protocols on patient perspectives such as quality of life, adherence and satisfaction. The manuscript management system is completely online and includes a very quick and fair peer-review system, which is all easy to use. Visit http://www.dovepress.com/ testimonials.php to read real quotes from published authors. 